\title{
Bioinformatics analysis of the prognostic value of CCT6A and associated signalling pathways in breast cancer
}

\author{
KAI HUANG ${ }^{1}$, YI ZENG ${ }^{1}$, YUNQING XIE ${ }^{2}$, LIYING HUANG ${ }^{1}$ and YU WU $^{3}$ \\ Departments of ${ }^{1}$ Breast Surgery, ${ }^{2}$ Surgical Laboratory and ${ }^{3}$ Head and Neck Surgery, Fujian Cancer Hospital, \\ Fujian Medical University Cancer Hospital, Fuzhou, Fujian 350001, P.R. China
}

Received July 23, 2018; Accepted February 20, 2019

DOI: $10.3892 / \mathrm{mmr} .2019 .10100$

\begin{abstract}
Breast cancer (BC) is the most frequently diagnosed type of cancer and the leading cause of cancer-associated mortality among women worldwide. However, the molecular basis for the pathogenesis of $\mathrm{BC}$ requires further exploration. Recent studies have demonstrated that chaperonin-containing TCP1 subunit 6A (CCT6A) efficiently suppresses transforming growth factor- $\beta$-mediated metastasis by inhibiting the function of SMAD family member 2 in lung cancer. However, the functional significance of CCT6A in other types of cancer, including $\mathrm{BC}$, remains to be investigated. Therefore, this study evaluated CCT6A expression in BC samples, and further analysed its association with survival, clinicopathological parameters and related signalling pathways using online datasets. The present study indicated that CCT6A expression was significantly higher in BC tissues compared with in surrounding noncancerous tissues at both mRNA and protein levels. Furthermore, increased CCT6A expression was significantly associated with poor survival, including overall survival, relapse-free survival, distant metastasis-free survival and post progression survival, in patients with BC. Pathway finder analysis indicated that CCT6A was significantly associated with the cell cycle, and its expression was significantly positively correlated with cyclin $(\mathrm{CCN}) \mathrm{B} 2$ and CCNA2 expression. Taken together, to the best of our knowledge, the present study is the first to indicate that CCT6A may serve a significant role in $\mathrm{BC}$ tumour progression.
\end{abstract}

\section{Introduction}

Breast cancer (BC) is the most frequently diagnosed type of cancer and the leading cause of cancer-associated mortality

Correspondence to: Dr Yu Wu, Department of Head and Neck Surgery, Fujian Cancer Hospital, Fujian Medical University Cancer Hospital, 420 Fuma Road, Fuzhou, Fujian 350001, P.R. China E-mail: headandneck2018@126.com

Key words: breast cancer, chaperonin-containing TCP1 subunit 6A, survival, cell cycle, pathway among women worldwide; 1.7 million new diagnoses and 521,900 cases of mortality are attributed to BC annually (1). However, despite extensive studies focussing on BC, and the development of novel and less toxic treatment regimens, the survival rates for patients with $\mathrm{BC}$ remain low (2). Therefore, the identification of more valuable and convenient biomarkers for early diagnosis and survival prediction, as well as novel therapeutic target genes for effective treatment, is urgently required.

Chaperonin-containing TCP1 subunit 6A (CCT6A) is the $\zeta$ subunit of the chaperonin-containing TCP1 complex (CCT), which comprises two identical rings stacked back to back, each containing eight different homologous proteins (1-8 or $\alpha-\theta)$; the protein encoded by CCT6A functions as a molecular chaperone (3-5). Previous studies have indicated that 5-10\% of all newly synthesised cytoplasmic proteins appear to pass this chaperone, suggesting it has an essential role in cytoskeletal organisation and in the cell cycle $(6,7)$. As a subunit of the CCT family, CCT6A is essential for the correct folding and oligomerisation/polymerisation of native proteins, with actin and tubulin being the major substrates (8). Furthermore, CCT6A has been identified as a specific component of endogenous extracellular signal-regulated kinase (ERK)1/2 signalling complexes (9) that possesses phosphorylation sites for mitogen-activated protein kinase (MAPK)/ERK $(10,11)$. In addition, CCT6A and the MAPK signalling pathway serve a critical role in matrix metalloproteinase-3-dependent regulation of neurite outgrowth and neuronal migration in the developing brain (7).

Previous studies have revealed that CCT6A expression is significantly increased in 10 human tumour cell lines and drug-resistant human melanoma cell lines, suggesting that CCT6A may have a critical role in tumorigenesis and drug resistance $(12,13)$. In addition, a recent experimental study on lung cancer indicated that CCT6A overexpression significantly increases colony and tumour sphere formation, as well as side population, and reduces sensitivity to anoikis in A549 cells following transforming growth factor (TGF)- $\beta$ stimulation (14). However, the functional significance of CCT6A in other types of cancer, including $\mathrm{BC}$, has yet to be investigated.

To determine the significance of CCT6A in BC, the present study evaluated CCT6A expression in various tumours, including BC, and analysed its association with survival, clinicopathological parameters and related signalling pathways 
using online datasets. The results revealed that various cancer tissues exhibited significantly higher CCT6A expression compared within noncancerous tissues, and increased CCT6A expression in BC tissues was significantly associated with poor patient prognosis. Furthermore, pathways based on associated genes indicated that CCT6A was significantly associated with the cell cycle, and its expression was significantly positively correlated with cyclin $(\mathrm{CCN}) \mathrm{B} 2$ and $\mathrm{CCNA} 2$ expression. Collectively, these findings suggested that CCT6A may have an important role in $\mathrm{BC}$ progression.

\section{Materials and methods}

Oncomine analysis. Gene expression array datasets from the online cancer microarray database Oncomine (www.oncomine.org) (15) were used to evaluate CCT6A mRNA expression in various tumour samples. CCT6A mRNA expression in $\mathrm{BC}$ tissues was compared with that in normal control tissues using 10 datasets from 3 cohorts $(16,17)$. The fold change in CCT6A expression from different datasets was presented using scatter plots. The datasets used in this study are listed in Table I. Details regarding standardised normalisation techniques and statistical calculations are provided on the Oncomine website (18). The search parameters and filters used to find the datasets were set as follows: P-value, 0.05; fold change, 1.5; gene rank, $10 \%$; analysis type, cancer vs. normal analysis; data type, mRNA.

Human Protein Atlas (HPA).CCT6A expression was furtherevaluated using the publicly available HPA (www.proteinatlas.org; version 18). The HPA is a database containing images of tissue microarrays labelled with antibodies against 11,250 human proteins (19). These tissue microarrays comprise sections from 46 normal human tissues and 20 types of human cancer. Analyses were performed using HPA images of breast sections labelled with CCT6A antibody (cat. no. HPA042996; Sigma-Aldrich; Merck KGaA, Darmstadt, Germany).

Kaplan-Meier plotter. The Kaplan-Meier plotter (www. kmplot.com) is an online database containing gene expression data and survival information from 4,142 clinical patients with BC $(20,21)$. To further investigate the prognostic value of CCT6A mRNA expression in BC, this study analysed the association between CCT6A mRNA expression and survival, including overall survival (OS), relapse-free survival (RFS), distant metastasis-free survival (DMFS) and post progression survival (PPS), in patients with BC. Samples from patients with $\mathrm{BC}$ were divided into two groups according to median CCT6A expression (high vs. low expression), after which the association between CCT6A mRNA expression and survival was analysed using a Kaplan-Meier survival plot together with hazard ratio, 95\% confidence interval and log-rank P-value.

Breast Cancer Gene-Expression Miner v4.0 (bc-GenExMiner v4.0). The bc-GenExMiner v4.0 (http://bcgenex.centregauducheau.fr/BC-GEM/GEM-Accueil.php?js=1) contains 36 annotated genomic datasets and three statistical mining functions $(22,23)$. The bc-GenExMiner v4.0 was used to compare target gene expression according to clinical criteria. The present study analysed the association between CCT6A expression and hormonal receptors, nodal status and other factors, in order to assess the prognostic impact of this candidate gene in human $\mathrm{BC}$ and to provide potential prognostic markers for $\mathrm{BC}$.

R2 application. R2: Genomics Analysis and Visualisation Platform (http://r2.amc.nl) (24) is a web-based genomics analysis and visualisation application, which was used to investigate CCT6A-related signalling pathways and genes. Pathway enrichment analysis based on CCT6A-associated genes was performed using the R2 Kyoto Encyclopedia of Genes and Genomes (KEGG) Pathway Finder and the standard setting in The Cancer Genome Atlas (TCGA) collection (BRCA was the TCGA ID and the search term in R2) (15). Furthermore, the correlation between CCT6A expression and related gene expression was calculated using the Pearson test based on the expression in $\mathrm{BC}$ cancer tissues and noncancerous normal tissues. The related genes were identified through the R2 Platform by searching 'Find Correlated genes with a single gene' or 'Correlate two genes'. The r-value, P-value and T-value were calculated and provided by $\mathrm{R} 2$.

Statistical analysis. The expression of CCT6A in BC tissues and noncancerous breast tissues was presented as the means $\pm 10 \%$ of percentile of the median intensity. Comparison of CCT6A expression between BC tissues and noncancerous breast tissues using the Oncomine data was performed using Student's t-test. The association between CCT6A mRNA expression and survival was analysed using Kaplan-Meier survival plots, which were statistically compared using the log-rank test on the Kaplan-Meier plotter website. Using the R2 website, the correlation between CCT6A expression and the expression of related genes was analysed using the Pearson test in BC samples, and the r-, P- and T-values were calculated and presented. The association between CCT6A and age, nodal status (+/-), receptor statuses [estrogen receptor $(\mathrm{ER})^{+}$vs. ER', progesterone receptor $(\mathrm{PR})^{+}$vs. $\mathrm{PR}^{-}$, human epidermal growth factor receptor $2(\mathrm{HER} 2)^{+}$vs. HER2 ${ }^{-}$] and triple-negative status was performed using a Welch's analysis of variance (ANOVA); if global significant differences were found (for correlation analysis of CCT6A with SBR and NPI grades), Dunnett-Tukey-Kramer's post-hoc test was performed to compare the differences among groups. $\mathrm{P}<0.05$ was considered to indicate a statistically significant difference.

\section{Results}

CCT6A upregulation in BC tissues. Analyses of mRNA expression in various tumour samples from the Oncomine platform indicated that CCT6A expression was significantly higher in tumour tissues compared with matched normal tissues (Fig. 1A). In particular, CCT6A was significantly upregulated in $\mathrm{BC}$ tissues compared with in noncancerous breast tissues (Fig. 1B-L; P<0.05). Details of CCT6A expression in these datasets are listed in Table I. Accordingly, as presented in Fig. 2, HPA (version 18) data analyses confirmed that BC tissues (images of T1-T3 availablefromhttps://www.proteinatlas.org/ENSG00000146731CCT6A/pathology/tissue/breast+cancer\#img) exhibited higher protein expression levels of CCT6A compared with normal 

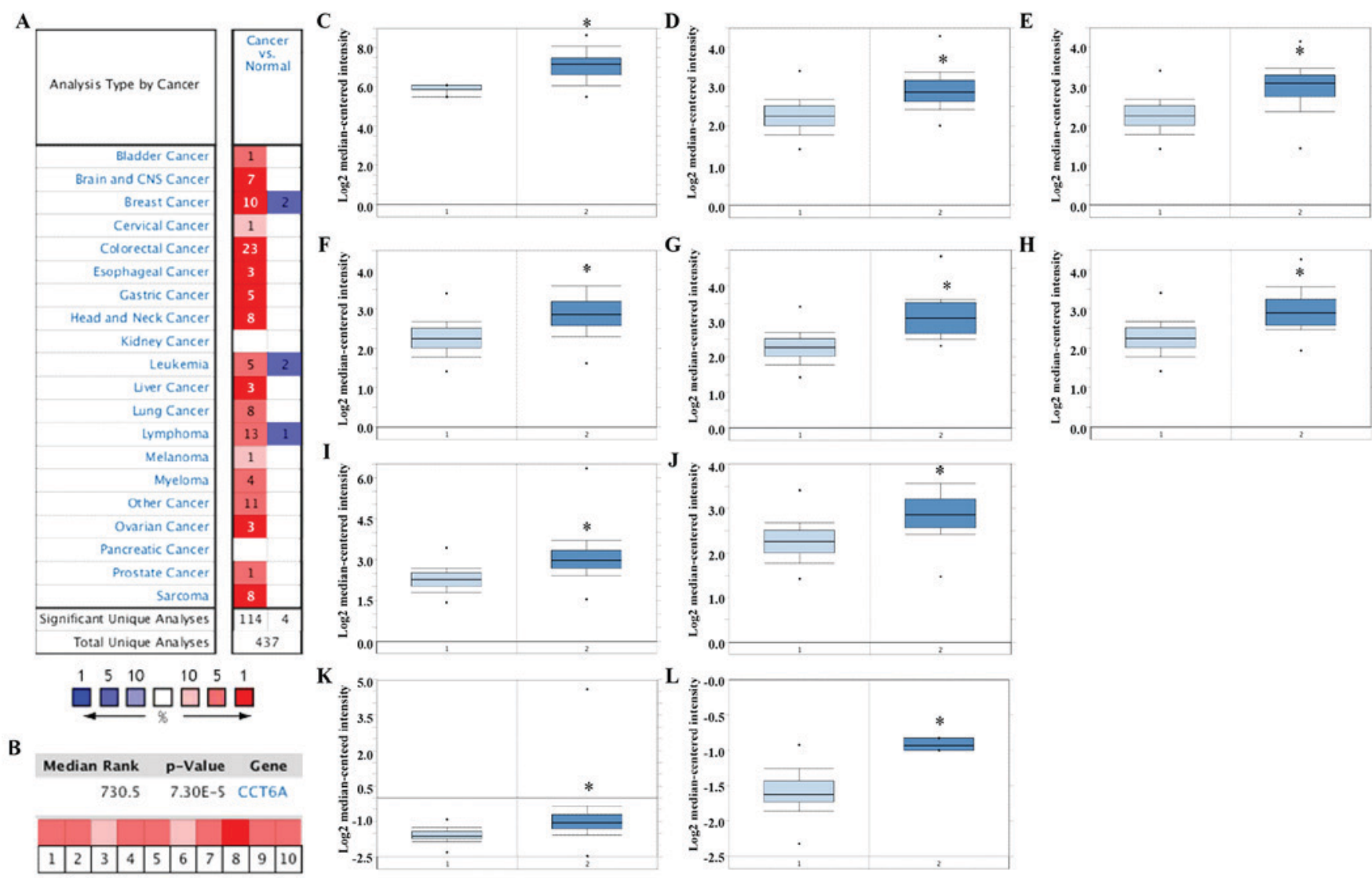

Figure 1. CCT6A mRNA expression in human breast cancer specimens. The mRNA expression levels of CCT6A in various cancer specimens were analysed using Oncomine. (A) Diagram showing the number of datasets with statistically significant $(\mathrm{P}<0.05)$ CCT6A mRNA overexpression (red) or downregulation (blue). (B) CCT6A was significantly upregulated in 10 breast cancer datasets. (C) Analysis from the 'Richardson Breast 2 Statistics' cohort. (D-J) Analyses from the 'Curtis Breast Statistics' cohort. (K-L) Analyses from the TCGA Breast Statistics. "P $<0.05$ vs. related noncancerous tissues. 1, noncancerous tissues; 2, breast cancer tissues; CCT6A, chaperonin-containing TCP1 subunit 6A.

N1

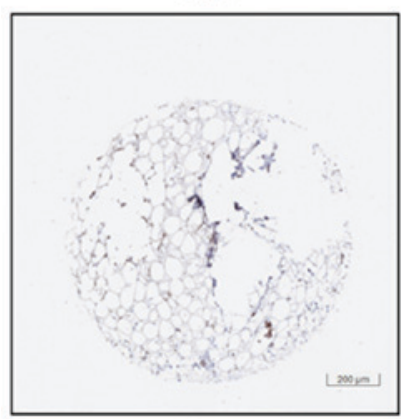

T1

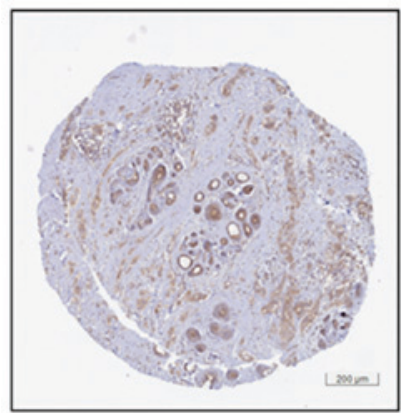

N2

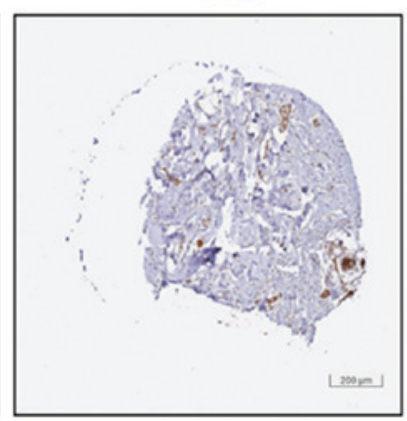

T2

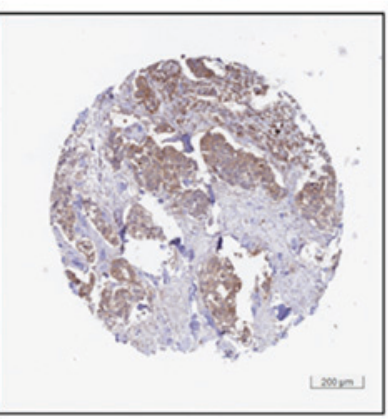

N3

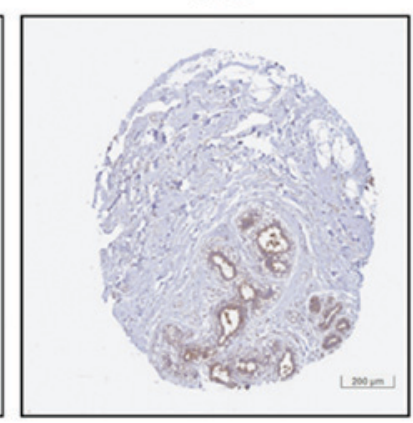

T3

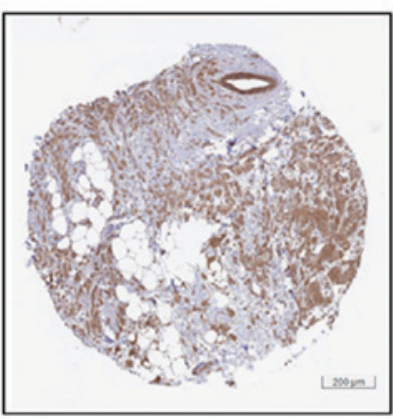

Figure 2. CCT6A protein expression in human $\mathrm{BC}$ specimens. CCT6A protein expression in $\mathrm{BC}$ and noncancerous breast tissues was analysed using the Human Protein Atlas (www.proteinatlas.org). Three representative images of BC and noncancerous breast tissues are presented. BC, breast cancer; CCT6A, chaperonin-containing TCP1 subunit 6A; N, normal; T, tumour. 


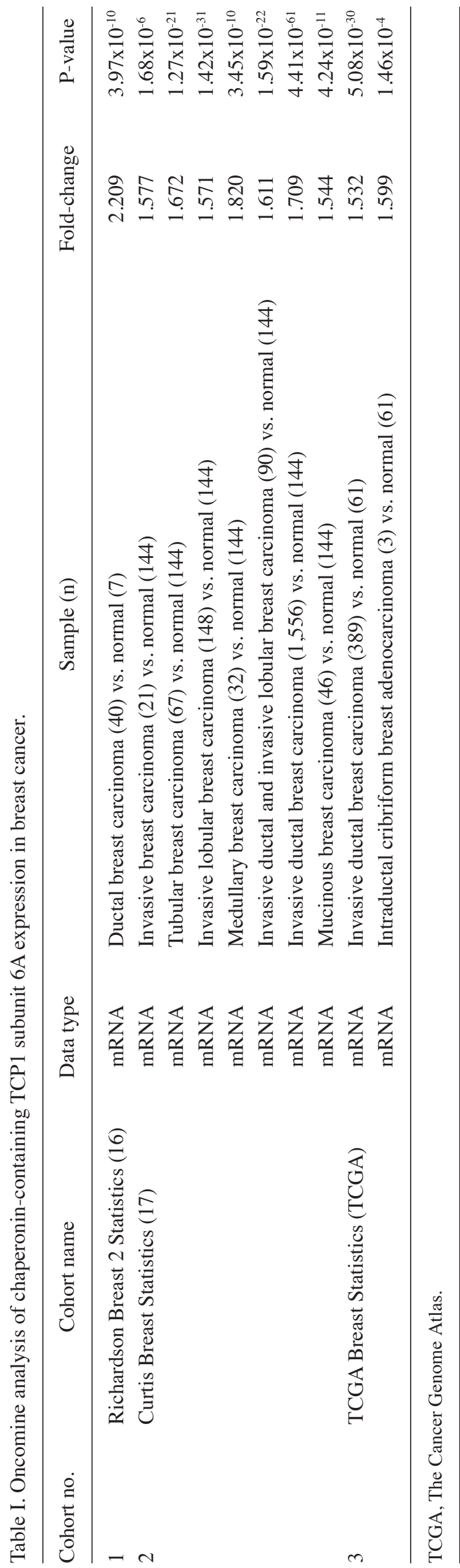

breast tissues (images of N1-N3 available from https://www. proteinatlas.org/ENSG00000146731-CCT6A/tissue/breast\#img).

Collectively, the aforementioned analyses demonstrated that the mRNA and protein expression levels of CCT6A were higher in $\mathrm{BC}$ tissues than in normal breast tissues, thus suggesting that CCT6A may act as an oncogene that promotes BC.

Association between CCT6A mRNA expression and clinicopathological parameters in patients with BC. Using Welch's ANOVA, CCT6A mRNA expression was compared among groups of patients with various clinicopathological parameters using bc-GenExMiner (Fig. 3 and Table II). In terms of age, CCT6A mRNA expression was lower in the older group ( $>51$ years; Fig. 3A; P=0.0007). Furthermore, among patients with $\mathrm{BC}$, those with positive nodal status exhibited higher CCT6A mRNA expression than those with negative nodal status (Fig. 3B; $\mathrm{P}=0.0259$ ). The $\mathrm{ER}$ and $\mathrm{PR}$ status of patients with BC was also negatively associated with CCT6A mRNA expression (Fig. 3C and D; $\mathrm{P}<0.0001$ ). However, there was no difference in CCT6A expression between patients with HER2 and HER2 ${ }^{+} \mathrm{BC}$ (Fig. 3E; $\mathrm{P}=0.0616$ ). Triple-negative $\mathrm{BC}$ (TNBC) is a type of $\mathrm{BC}$ characterised by ER', $\mathrm{PR}^{-}$and HER2CCT6A mRNA expression was significantly upregulated in patients with TNBC (Fig. 3F; $\mathrm{P}<0.0001$ ). The results also indicated that patients with basal-like BC exhibited higher CCT6A mRNA expression than those without basal-like BC (Fig. 3G; $\mathrm{P}<0.0001)$. With regards to Scarff-Bloom-Richardson (SBR) grading (25), a more advanced SBR grade was significantly associated with higher CCT6A mRNA expression (Fig. 3H; $\mathrm{P}<0.0001)$ compared with other SBR grades. Furthermore, a more advanced Nottingham prognostic index (NPI) grade (26) was associated with higher CCT6A mRNA expression (Fig. 3I; $\mathrm{P}<0.0001)$ compared with other NPI grades.

Higher CCT6A expression is associated with poor survival in patients with $B C$. Using the Kaplan-Meier plotter, the association between CCT6A expression and survival in patients with BC was evaluated. Kaplan-Meier analysis revealed that high CCT6A expression (Affymetrix microarrays, 201326-at and 201327_s_at; Fig. 4A and B) in BC tissues was significantly associated with shorter OS $(\mathrm{P}<0.05)$, RFS $(\mathrm{P}<0.05)$, DMFS $(\mathrm{P}<0.05)$ and PPS $(\mathrm{P}<0.05)$ in patients with $\mathrm{BC}$, thus suggesting that high CCT6A expression may be predictive of poor prognosis.

Possible involvement of CCT6A in the cell cycle and its positive correlation with CCNB2 and CCNA2. To further investigate the signalling pathways in which CCT6A might be involved, pathway enrichment analysis was performed using the R2 KEGG Pathway Finder based on CCT6A-associated genes in $\mathrm{BC}$ tissues from TCGA dataset (ID: BRCA). Accordingly, the top 10 signalling pathways associated with CCT6A, including the 'cell cycle', are presented in Fig. 5 and Table III. Among these correlated genes involved in the cell cycle, CCT6A expression was significantly positively correlated with CCNB2 (r-value $=0.560 ; \mathrm{P}=1.0 \times 10^{-91} ; \mathrm{T}$-value $\left.=22.389\right)$ and CCNA2 (r-value $=0.532 ; \mathrm{P}=2.7 \times 10^{-81} ; \mathrm{T}$-value $\left.=20.807\right)$ expression . These results indicated that CCT6A may be involved in the cell cycle signalling pathway. 
A

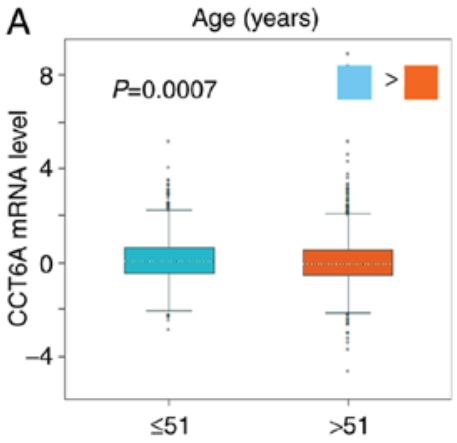

D

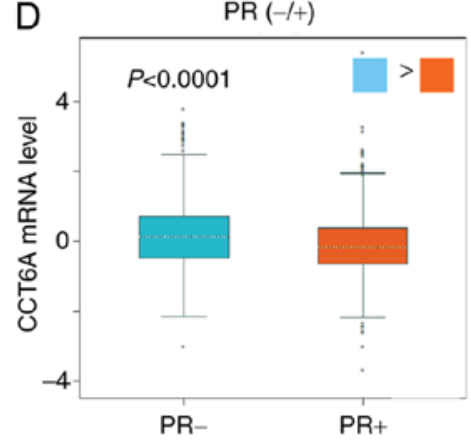

G



B



E

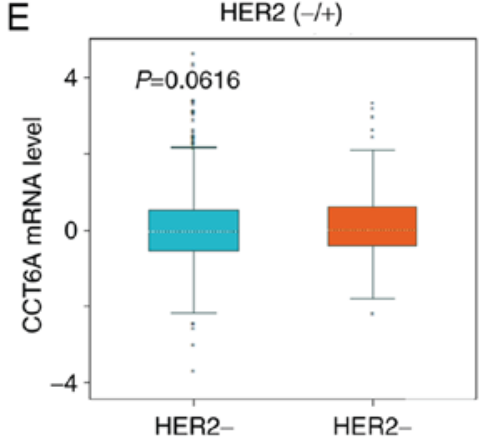

$\mathrm{H}$

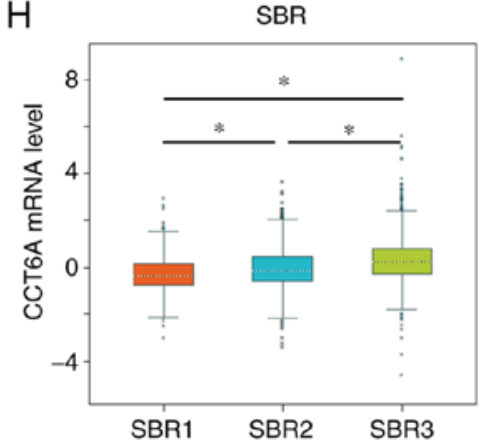

C



$\mathrm{F}$

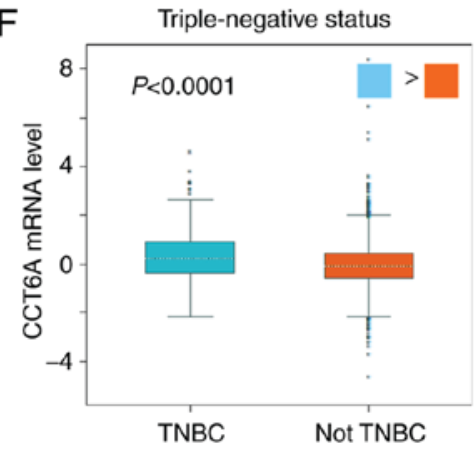

I

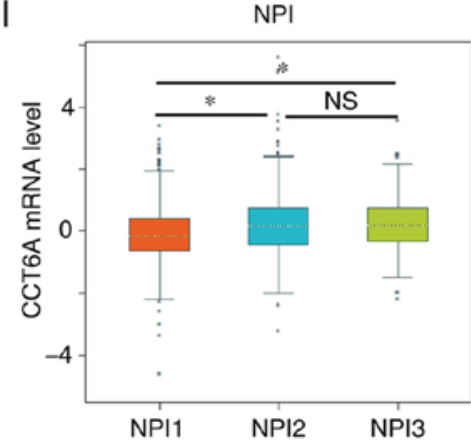

Figure 3. Association between CCT6A mRNA expression and clinicopathological parameters in patients with BC. Association between CCT6A mRNA expression and (A) age, (B) nodal status, (C) ER status, (D) PR status, (E) HER2 status, (F) TNBC, (G) basal-like status, (H) SBR grade and (I) NPI grade. ${ }^{*} \mathrm{P}<0.05$, as analysed using Breast Cancer Gene-Expression Miner v4.0. BC, breast cancer; CCT6A, chaperonin-containing TCP1 subunit 6A; ER, estrogen receptor; HER2, human epidermal growth factor receptor 2; IHC, immunohistochemistry; NPI, Nottingham prognostic index; NS, not significant; SBR, Scarff-Bloom-Richardson; TNBC, triple-negative breast cancer.

\section{Discussion}

$\mathrm{BC}$ is the most common malignant tumour among women worldwide (1), and has been reported to be associated with the overexpression of oncogenes (27-29). Despite significant improvements in the diagnosis, treatment and survival prediction of $\mathrm{BC}$, the identification of novel biomarkers for prognosis is urgently required. Studies have revealed that CCT6A expression is increased in 10 human tumour cell lines and drug-resistant human melanoma cell lines $(12,13)$. However, to the best of our knowledge, its expression in clinical tumour samples has not been evaluated previously. Using the online Oncomine dataset, the present study demonstrated that the majority of tumour tissues, including breast, lung, liver and colorectal cancer tissues, exhibited significantly higher CCT6A expression compared within adjacent noncancerous tissues. In the majority of the $\mathrm{BC}$ datasets (10/12), BC tissues exhibited significantly higher CCT6A mRNA expression compared with in noncancerous breast tissues; the same observation was confirmed at the protein level by immunohistochemistry (IHC) through HPA. These results indicated that CCT6A may be among the overexpressed genes in tumour tissues and may serve a critical role in tumorigenesis. However, the protein expression levels of CCT6A in BC samples should be further validated via western blot analysis and immunohistochemistry.

It has previously been reported that increased CCT6A expression is significantly associated with poor survival in patients who have high TGF- $\beta$ levels in lung cancer tissues, but not in those with low TGF- $\beta$ levels, suggesting that CCT6A serves as a valuable biomarker for lung cancer (14). Using the Kaplan-Meier plotter, it was demonstrated that high CCT6A expression was significantly associated with unfavourable OS, RFS, DMFS and PPS in all patients with BC, indicating that CCT6A mRNA expression may serve as a prognostic indicator in patients with BC. In addition, CCT6A overexpression in cancer cells significantly shortens the survival time in tumour-bearing mice, whereas CCT6A knockdown significantly prolongs survival (14). Taken together, these observations further support the role of CCT6A as an essential biomarker for survival prediction in patients with cancer, including BC. 
Table II. Association between mRNA expression of chaperonin-containing TCP1 subunit 6Aand clinicopathological parameters of breast cancer.

\begin{tabular}{|c|c|c|c|}
\hline Variable & Case & mRNA expression & $\mathrm{P}$-value \\
\hline Age (years) & & & 0.0007 \\
\hline$\leq 51$ & 1,392 & $\uparrow$ & \\
\hline$<51$ & 2,210 & - & \\
\hline Nodal status & & & 0.0259 \\
\hline Negative & 2,493 & - & \\
\hline Positive & 1,562 & $\uparrow$ & \\
\hline ER (IHC) & & & $<0.0001$ \\
\hline Negative & 1,446 & $\uparrow$ & \\
\hline Positive & 3,766 & - & \\
\hline PR (IHC) & & & $<0.0001$ \\
\hline Negative & 804 & $\uparrow$ & \\
\hline Positive & 1,249 & - & \\
\hline HER2 (IHC) & & & 0.0616 \\
\hline Negative & 1,409 & - & \\
\hline Positive & 201 & - & \\
\hline Triple-negative status & & & $<0.0001$ \\
\hline Not TNBC & 374 & $\uparrow$ & \\
\hline TNBC & 3,857 & - & \\
\hline Basal-like status & & & $<0.0001$ \\
\hline Basal-like & 1,060 & $\uparrow$ & \\
\hline Not basal-like & 3,942 & - & \\
\hline SBR grade & & & $<0.0001$ \\
\hline SBR1 & 546 & - & \\
\hline SBR2 & 1,431 & $\uparrow$ & \\
\hline SBR3 & 1,317 & $\uparrow$ & \\
\hline NPI grade & & & $<0.0001$ \\
\hline NPI1 & 931 & - & \\
\hline NPI2 & 727 & $\uparrow$ & \\
\hline NPI3 & 125 & $\uparrow$ & \\
\hline
\end{tabular}

ER, estrogen receptor; HER2, human epidermal growth factor receptor 2; IHC, immunohistochemistry; NPI, Nottingham prognostic index; SBR, Scarff-Bloom-Richardson; TNBC, triple-negative breast cancer.

Table III. Pathway enrichment analysis of chaperonin-containing TCP1 subunit 6A-associated genes in TCGA.

\begin{tabular}{rlcccc}
\hline No. & \multicolumn{1}{c}{ Pathway } & No. of input genes & Total no. of genes & Percentage (\%) & P-value \\
\hline 1 & Cell_cycle & 108 & 124 & 87.10 & $9.50 \times 10^{-5}$ \\
2 & Fanconi_anemia_pathway & 49 & 52 & 94.20 & $2.50 \times 10^{-4}$ \\
3 & Pyrimidine_metabolism & 88 & 102 & 86.30 & $7.90 \times 10^{-4}$ \\
4 & Proteasome & 41 & 44 & 93.20 & $1.30 \times 10^{-3}$ \\
5 & Pathways_in_cancer & 311 & 397 & 78.30 & $1.80 \times 10^{-3}$ \\
6 & DNA_replication & 34 & 36 & 94.40 & $2.10 \times 10^{-3}$ \\
7 & Spliceosome & 109 & 131 & 83.20 & $2.50 \times 10^{-3}$ \\
8 & Ribosome_biogenesis_in_eukaryotes & 66 & 76 & 86.80 & $2.60 \times 10^{-3}$ \\
9 & MicroRNAs_in_cancer & 122 & 149 & 81.90 & $4.10 \times 10^{-3}$ \\
10 & Glutamatergic_synapse & 94 & 113 & 83.20 & $5.00 \times 10^{-3}$ \\
\hline
\end{tabular}


A
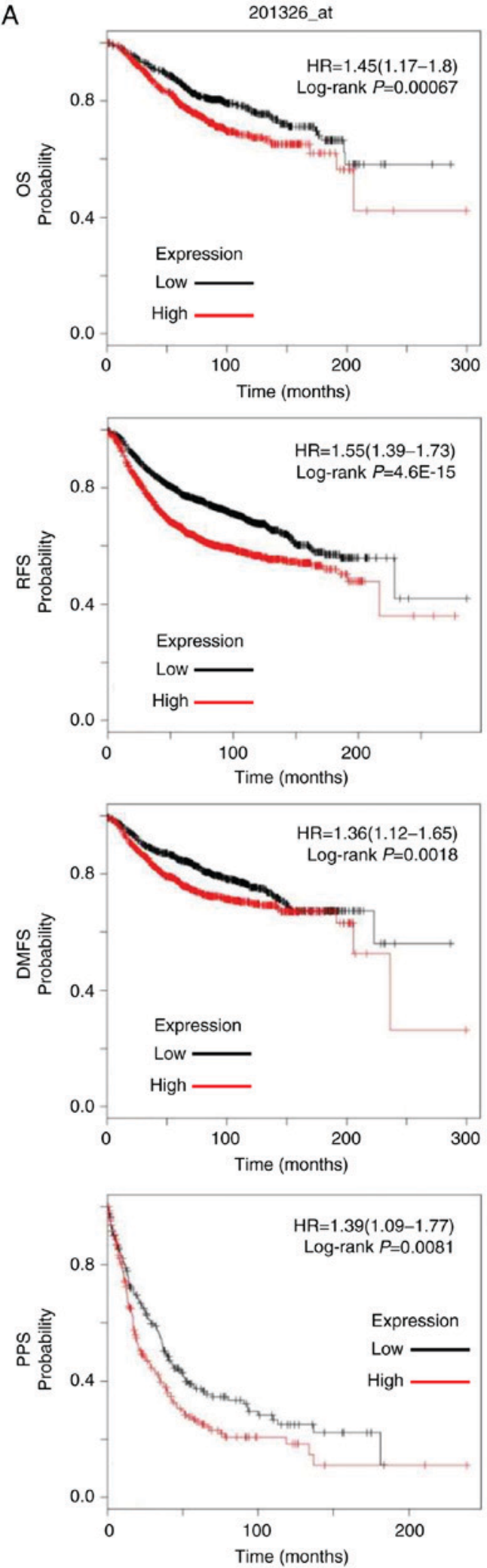

B
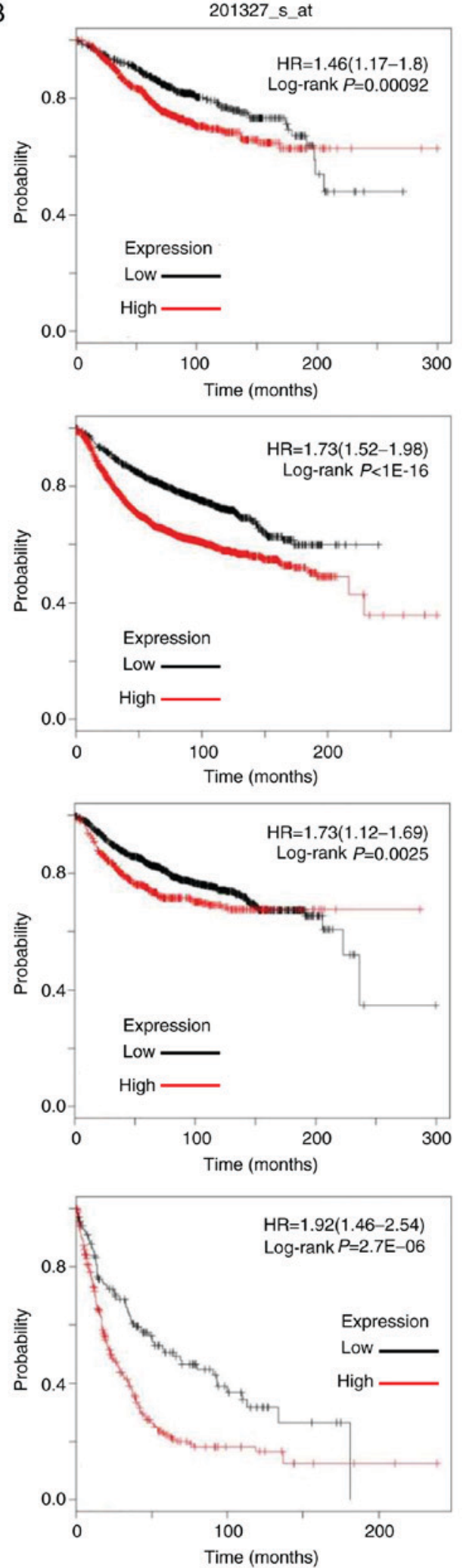

Figure 4. Association between CCT6A expression and survival in patients with BC. Association between CCT6A (A) 201326_at and (B) 201327_s_at expression and OS, RFS, DMFS and PPS in patients with BC was analysed using log-rank tests based on CCT6A expression in BC tissues from The Cancer Genome Atlas dataset. Kaplan-Meier curves were plotted for CCT6A, and HRs and 95\% confidence intervals are shown. BC, breast cancer; CCT6A, chaperonin-containing TCP1 subunit 6A; DMFS, distant metastasis-free survival; HR, hazard ratio; OS, overall survival; RFS, disease-free survival; PPS, post progression survival.

$\mathrm{BC}$ is classified into distinct histological and biological subtypes with different pathological, molecular and clinical features (30). Therefore, this study further investigated the association between CCT6A expression, at both the mRNA and protein levels, and clinicopathological parameters in patients with BC. The findings indicated that high CCT6A expression was negatively associated with ER and PR status, and positively associated with nodal, basal-like and TNBC status. 



Figure 5. CCT6A is involved in the 'cell cycle' pathway and correlated genes. (A) Pathway Finder was used to analyse pathways based on CCT6A-associated genes from TCGA dataset. (B and C) Correlation between CCT6A expression and CCNA2 and CCNB2 expression was analysed based on their expression in BC samples from TCGA dataset. BC, breast cancer; CCN, cyclin; CCT6A, chaperonin-containing TCP1 subunit 6A; TCGA, The Cancer Genome Atlas.

Furthermore, higher SBR and NPI grades were significantly associated with higher CCT6A expression. HER2 is a member of the epidermal growth factor receptor family (31), and is associated with poor outcome in patients with BC. However, this study found no association between CCT6A expression and HER 2 status. Taken together, these findings demonstrated that increased CCT6A expression may be significantly associated with poor outcomes in patients with $\mathrm{BC}$, while possibly serving as a novel biomarker for the prognosis of $\mathrm{BC}$.

The evaluation of biological functions has revealed that CCT6A overexpression significantly increases colony and tumour sphere formation, increases side population and reduces sensitivity to anoikis in A549 cells following TGF- $\beta$ stimulation (14). However, to the best of our knowledge, the biological functions and underlying mechanism of CCT6A in $\mathrm{BC}$ remain to be evaluated. Accordingly, following the analyses of signalling pathways associated with CCT6A based on correlated genes, it was demonstrated that 'cell cycle' was among the most enriched KEGG signalling pathways. In addition, CCT6A expression was positively correlated with the expression of CCNB2 and CCNA2, two important cell cycle regulators, thus suggesting that CCT6A may be involved in tumour growth mediated by the cell cycle. However, further investigations on the biological function and underlying mechanism of action of CCT6A in BC are required. Future studies should aim to assess the role of CCT6A in growth, metastasis and chemotherapeutic resistance in cancer, including BC, which may improve understanding of the role of CCT6A in the progression of cancer.

In conclusion, the present study revealed that $\mathrm{BC}$ tissues exhibited significantly higher CCT6A expression compared with in noncancerous breast tissues at the mRNA and protein levels. In addition, increased CCT6A expression was significantly associated with unfavourable survival in patients with BC. Furthermore, CCT6A expression was negatively associated with ER and PR status, positively correlated with nodal, basal-like and TNBC status, and was increased in patients with advanced SBR and NPI grades. CCT6A was also revealed to be involved in the cell cycle, and its expression was positively correlated with CCNB2 and CCNA2 expression. Collectively, CCT6A may be a potential target in BC.

\section{Acknowledgements}

Not applicable.

\section{Funding}

No funding was received.

\section{Availability of data and materials}

The datasets used and/or analysed during the current study are available from the corresponding author 
on reasonable request. The images presented in Fig. 2 were provided by the Human Protein Atlas: BC tissues (https:/www.proteinatlas.org/ENSG00000146731-CCT6A/pathology/ tissue/breast+cancer\#img); normal breast tissues (https://www. proteinatlas.org/ENSG00000146731-CCT6A/tissue/breast\#img).

\section{Authors' contributions}

$\mathrm{KH}$ and YW conceived and designed the experiments. $\mathrm{KH}$ and YZ performed the Oncomine analysis. YX and LH conducted the HPA analysis. YZ and YX conducted the Breast Cancer Gene-Expression Miner analysis. LH and YX conducted the survival analysis. YW conducted the R2 KEGG Pathway Finder analysis. KH and YW wrote the manuscript. All authors read and approved the final manuscript.

\section{Ethics approval and consent to participate}

Not applicable.

\section{Patient consent for publication}

Not applicable.

\section{Competing interests}

The authors declare that they have no competing interests.

\section{References}

1. Siegel RL, Miller KD and Jemal A: Cancer statistics, 2017. CA Cancer J Clin 67: 7-30, 2017

2. Yu Y, Walia V and Elble RC: Loss of CLCA4 promotes epithelial-to-mesenchymal transition in breast cancer cells. PLoS One 8: e83943, 2013.

3. Hynes G, Kubota H and Willison KR: Antibody characterisation of two distinct conformations of the chaperonin-containing TCP-1 from mouse testis. FEBS Lett 358: 129-132, 1995.

4. Kubota H, Hynes G, Carne A, Ashworth A and Willison K Identification of six Tcp-1-related genes encoding divergent subunits of the TCP-1-containing chaperonin. Curr Biol 4: 89-99, 1994

5. Rommelaere H, Van Troys M, Gao Y, Melki R, Cowan NJ, Vandekerckhove J and Ampe C: Eukaryotic cytosolic chaperonin contains t-complex polypeptide 1 and seven related subunits. Proc Natl Acad Sci USA 90: 11975-11979, 1993.

6. Yam AY, Xia Y, Lin HT, Burlingame A, Gerstein M and Frydman J: Defining the TRiC/CCT interactome links chaperonin function to stabilization of newly made proteins with complex topologies. Nat Struct Mol Biol 15: 1255-1262, 2008.

7. Van Hove I, Verslegers M, Hu TT, Carden M, Arckens L and Moons L: A proteomic approach to understand MMP-3-driven developmental processes in the postnatal cerebellum: Chaperonin CCT6A and MAP kinase as contributing factors. Dev Neurobiol 75: 1033-1048, 2015.

8. Brackley KI and Grantham J: Activities of the chaperonin containing TCP-1 (CCT): Implications for cell cycle progression and cytoskeletal organisation. Cell Stress Chaperones 14 23-31, 2009.

9. von Kriegsheim A, Baiocchi D, Birtwistle M, Sumpton D, Bienvenut W, Morrice N, Yamada K, Lamond A, Kalna G, Orton R, et al: Cell fate decisions are specified by the dynamic ERK interactome. Nat Cell Biol 11: 1458-1464, 2009.

10. Xiang B, Chatti K, Qiu H, Lakshmi B, Krasnitz A, Hicks J, Yu M, Miller WT and Muthuswamy SK: Brk is coamplified with ErbB2 to promote proliferation in breast cancer. Proc Natl Acad Sci USA 105: 12463-12468, 2008.

11. Roskoski R Jr: ERK1/2 MAP kinases: Structure, function, and regulation. Pharmacol Res 66: 105-143, 2012.
12. Tanic N, Brkic G, Dimitrijevic B, Dedovic-Tanic N, Gefen N, Benharroch D and Gopas J: Identification of differentially expressed mRNA transcripts in drug-resistant versus parental human melanoma cell lines. Anticancer Res 26: 2137-2142, 2006.

13. Myung JK, Afjehi-Sadat L, Felizardo-Cabatic M, Slave I, Lubec G: Expressional patterns of chaperones in ten human tumor cell lines. Proteome Sci 2: 8, 2004.

14. Ying Z, Tian H, Li Y, Lian R, Li W, Wu S, Zhang HZ, Wu J, Liu L, Song J, et al: CCT6A suppresses SMAD2 and promotes prometastatic TGF- $\beta$ signaling. J Clin Invest 127: 1725-1740, 2017.

15. Rhodes DR, Yu J, Shanker K, Deshpande N, Varambally R, Ghosh D, Barrette T, Pandey A and Chinnaiyan AM: ONCOMINE: A cancer microarray database and integrateddata-mining platform. Neoplasia 6: 1-6, 2004.

16. Richardson AL, Wang ZC, De Nicolo A, Lu X, Brown M, Miron A, Liao X, Iglehart JD, Livingston DM and Ganesan S: X chromosomal abnormalities in basal-like human breast cancer. Cancer Cell 9: 121-132, 2006

17. Curtis C, Shah SP, Chin SF, Turashvili G, Rueda OM, Dunning MJ, Speed D, Lynch AG, Samarajiwa S, Yuan Y, et al: The genomic and transcriptomic architecture of 2,000 breast tumours reveals novel subgroups. Nature 486: 346-352, 2012.

18. Shin S, Kim Y, Chul Oh S, Yu N, Lee ST, Rak Choi J and Lee KA: Validation and optimization of the Ion Torrent S5 XL sequencer and Oncomine workflow for BRCA1 and BRCA2 genetic testing. Oncotarget 8: 34858-34866, 2017.

19. Uhlen M, Oksvold P, Fagerberg L, Lundberg E, Jonasson K, Forsberg M,Zwahlen M,Kampf C, Wester K,Hober S, et al: Towards a knowledge-based Human Protein Atlas. Nat Biotechnol 28: $1248-1250,2010$

20. Győrffy B, Surowiak P, Budczies J and Lánczky A: Online survival analysis software to assess the prognostic value of biomarkers using transcriptomic data in non-small-cell lung cancer. PLoS One 8: e82241, 2013

21. Györffy B, Lanczky A, Eklund AC, Denkert C, Budczies J, Li Q and Szallasi Z: An online survival analysis tool to rapidly assess the effect of 22,277 genes on breast cancer prognosis using microarray data of 1,809 patients. Breast Cancer Res Treat 123: 725-731, 2010.

22. Jézéquel P, Campone M, Gouraud W, Guérin-Charbonnel C, Leux C, Ricolleau G and Campion L: bc-GenExMiner: An easy-to-use online platform for gene prognostic analyses in breast cancer. Breast Cancer Res Treat 131: 765-775, 2012.

23. Jézéquel P, Frénel JS, Campion L, Guérin-Charbonnel C, Gouraud W, Ricolleau G and Campone M: bc-GenExMiner 3.0: New mining module computes breast cancer gene expression correlation analyses. Database (Oxford) 2013: bas060, 2013.

24. Yu Y, Shang R, Chen Y, Li J, Liang Z, Hu J, Liu K and Chen C: Tumor suppressive ZBTB4 inhibits cell growth by regulating cell cycle progression and apoptosis in Ewing sarcoma. Biomed Pharmacother 100: 108-115, 2018.

25. Le Doussal V, Tubiana-Hulin M, Friedman S, Hacene K, Spyratos F and Brunet M: Prognostic value of histologic grade nuclear components of Scarff-Bloom-Richardson (SBR). An improved score modification based on a multivariate analysis of 1262 invasive ductal breast carcinomas. Cancer 64: 1914-1921, 1989.

26. Galea MH, Blamey RW, Elston CW and Ellis IO: The Nottingham prognostic index in primary breast cancer. Breast Cancer Res Treat 22: 207-219, 1992.

27. Li C, Du L, Ren Y, Liu X, Jiao Q, Cui D, Wen M, Wang C, Wei G, Wang Y, et al: SKP2 promotes breast cancer tumorigenesis and radiation tolerance through PDCD4 ubiquitination. J Exp Clin Cancer Res 38: 76, 2019.

28. Pucci S, Polidoro C, Greggi C, Amati F, Morini E, Murdocca M, Biancolella M, Orlandi A, Sangiuolo F and Novelli G: Pro-oncogenic action of LOX-1 and its splice variant LOX-1 $\Delta 4$ in breast cancer phenotypes. Cell Death Dis 10: 53, 2019.

29. O'Toole SA, Selinger CI, Millar EK, Lum T and Beith JM: Molecular assays in breast cancer pathology. Pathology 43: 116-127, 2011.

30. Michaut M, Chin SF, Majewski I, Severson TM, Bismeijer T, de Koning L, Peeters JK, Schouten PC, Rueda OM, Bosma AJ, et al: Integration of genomic, transcriptomic and proteomic data identifies two biologically distinctsubtypes of invasive lobular breast cancer. Sci Rep 6: 18517, 2016.

31. Slamon DJ, Clark GM, Wong SG, Levin WJ, Ullrich A and McGuireWL:Humanbreastcancer:Correlationofrelapseandsurvival with amplification of the HER-2/neu oncogene. Science 235: 177-182, 1987.

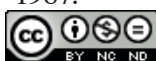

This work is licensed under a Creative Commons Attribution-NonCommercial-NoDerivatives 4.0 International (CC BY-NC-ND 4.0) License. 